AperTO - Archivio Istituzionale Open Access dell'Università di Torino

\title{
Muscle Synergy Assessment during Single-Leg Stance
}

\section{This is the author's manuscript}

Original Citation:

\section{Availability:}

This version is available http://hdl.handle.net/2318/1762210

since 2020-11-10T12:13:07Z

Published version:

DOI:10.1109/TNSRE.2020.3030847

Terms of use:

Open Access

Anyone can freely access the full text of works made available as "Open Access". Works made available under a Creative Commons license can be used according to the terms and conditions of said license. Use of all other works requires consent of the right holder (author or publisher) if not exempted from copyright protection by the applicable law. 


\title{
Muscle Synergy Assessment during Single-Leg Stance
}

\author{
M. Ghislieri, Student Member, IEEE, M. Knaflitz, Member, IEEE, L. Labanca, G. Barone, L. \\ Bragonzoni, M. G. Benedetti, and V. Agostini, Member, IEEE
}

\begin{abstract}
In the study of muscle synergies during the maintenance of single-leg stance there are several methodological issues that must be taken into account before muscle synergy extraction. In particular, it is important to distinguish between epochs of surface electromyography (sEMG) signals corresponding to "well-balanced" and "unbalanced" single-leg stance, since different motor control strategies could be used to maintain balance. The aim of this work is to present and define a robust procedure to distinguish between "well-balanced" and "unbalanced" single-leg stance to be chosen as input for the algorithm used to extract muscle synergies. Our results demonstrate that the proposed approach for the selection of sEMG epochs relative to "well-balanced" and "unbalanced" single-leg stance is robust with respect to the selection of the segmentation threshold, revealing a high consistency in the number of muscle synergies and high similarity among the weight vectors (correlation values range from 0.75 to 0.97 ). Moreover, differences in terms of average recruitment levels and balance control strategies were detected, suggesting a slightly different modular organization between "well-balanced" and "unbalanced" single-leg stance. In conclusion, this approach can be successfully used as a pre-processing step before muscle synergy extraction, allowing for a better assessment of motor control strategies during the singleleg stance task.
\end{abstract}

Index Terms-balance, EMG, motor control, motor modules, unipedal stance.

\section{INTRODUCTION}

$\mathrm{T}$ HE study of human balance in upright stance is useful to test motor skills and evaluate proprioception and coordination, with applications to both clinics and sport. The postural sway can be studied in various balance tasks, such as single-leg stance (SLS), tandem, semi-tandem, and double-leg stance (DLS) [1]. Different motor control strategies and levels of difficulty in carrying out the task characterize these balance tasks. In particular, the unipedal stance or SLS requires the maintenance of postural stability standing on a single limb, and it may be challenging in subjects affected by chronic ankle instability (CAI) [2]-[4]. Considering a specific balance exercise, different conditions of visual and somatosensory integrations may be tested [5]. Typically, along with the eyes

M. Ghislieri, M. Knaflitz, and V. Agostini are with the Department of Electronics and Telecommunications of Politecnico di Torino and with PoliTo ${ }^{\mathrm{BIO}}$ MedLab of Politecnico di Torino, Turin, 10129, Italy. (e-mail: marco.ghislieri@polito.it, valentina.agostini@polito.it). open (EO) condition, in which the subject exploits the visual feedback to maintain balance, a condition with eyes closed (EC) is also studied to evaluate the effect of visual deprivation on postural balance control [6]-[8].

Recently, the assessment of motor control strategies during different motor tasks, such as postural balance or gait, has been studied by means of the muscle synergy theory [9]-[13]. According to this theory, our Central Nervous System (CNS) controls specific groups of muscles (muscle synergies) to perform the motor task, rather than control every single muscle involved. The main fields of application of muscle synergies are not only in clinics (e.g., neurorehabilitation), but also in bipedal robotics and sport [14]. Muscle synergies are usually extracted from surface electromyographic (sEMG) signals through data reduction algorithms. The most used algorithm to extract muscle synergies is Non-Negative Matrix Factorization (NNMF) [15], [16].

The study of muscle synergies is facing new challenges in the field of postural balance analysis. In literature, the study of muscle synergies in upright stance is mainly focused on the evaluation of balance recovery after a perturbation [17]-[21]. In particular, it was demonstrated that muscle synergies are highly consistent across different balance tasks [19], [22], [23]. This suggests that, increasing the task complexity, there should be only slight modifications to the basic motor control strategies involved in postural balance control. However, to the best of our knowledge, no studies are focusing on the muscle synergies adopted to maintain SLS. One possible reason may be the difficulty to select epochs of sEMG signals when the subject firmly maintains unipedal stance. Indeed, it is important to separate sEMG epochs in which balance is properly maintained from those in which a slight disequilibrium occurs. Therefore, this work aims at defining a robust procedure to distinguish between epochs of sEMG signals, relative to a "well-balanced" (WB) and "unbalanced" (UB) SLS. These signal epochs will be used as separate inputs for the muscle synergy extraction algorithm. The comparison of EC/EO conditions will be also introduced for completeness. The proposed approach might help the interpretation of muscle synergies in the SLS task.

L. Labanca and M. G. Benedetti are with the Physical Medicine and Rehabilitation Unit (IRCCS) of Istituto Ortopedico Rizzoli, Bologna, 40136, Italy. (e-mail: luciana.labanca88@gmail.com, mariagrazia.benedetti@ior.it).

G. Barone and L. Bragonzoni are with the Department for Life Quality Studies of the University of Bologna, Bologna, 40136, Italy. (e-mail: giuseppebarone91@gmail.com, 1.bragonzoni@biomec.ior.it). 


\section{MATERIALS AND MethodS}

\section{A. Sample Population}

Twenty-two healthy subjects (11 females and 11 males; age: $24 \pm 3$ years; height: $175.7 \pm 9.6 \mathrm{~cm}$; weight: $65.9 \pm 12.2 \mathrm{~kg}$ ) were enrolled in the study. None of the enrolled volunteers reported lower limb injuries or had neurological or musculoskeletal disorders that could compromise the execution of SLS. All the subjects were right-limb dominant, according to the preferred lower limb to start walking. This study was reviewed and approved by the Ethics Committee of the Area Vasta Emilia Centro della Regione Emilia Romagna (CE AVEC 193/2019/Sper/IOR approved on October 4, 2019). All participants signed written informed consent for the experimental procedure, and all the acquisitions were performed in accordance with the Declaration of Helsinki.

\section{B. Experimental Protocol}

The volunteers were asked to perform a SLS task for 2 times, once with eyes open (EO) and once with eyes closed (EC), randomizing the order condition for each subject. More specifically, in each test, the subject performed a transition from double-leg stance (DLS) to single-leg stance (SLS), maintaining SLS for at least 30 seconds, and then returning back to DLS. The test was performed on a firm surface (force plate), with the subject keeping the arms straight at the sides. In the EC condition, the subject closed the eyes right after reaching the SLS balance. If the subject failed to maintain the SLS balance for at least $30 \mathrm{~s}$ and required to land on both feet during the task, the test was stopped and repeated a second time. Figure 1 represents the block diagram of the experimental protocol.

\section{Data Acquisitions}

During the experimental protocol, the following signals were simultaneously recorded:

i. sEMG signals through active probes (FREEEMG 1000, BTS Bioengineering, Milan, Italy)

ii. Foot-switch signal to detect the onset/offset timing of the SLS (FREEEMG 1000 - Footswitch Kit, BTS Bioengineering, Milan, Italy)

iii. Ground reaction force by mean of a force plate (Dynamic Walkway P6000, BTS Bioengineering, Milan, Italy).

The sEMG signals were acquired from 13 muscles:

- 2 muscles of the trunk: right Longissimus Dorsii $\left(\mathrm{LD}_{\mathrm{R}}\right)$, and left Longissimus Dorsii $\left(\mathrm{LD}_{\mathrm{L}}\right)$

- 11 muscles of the dominant (right) lower limb: Gluteus Medius (GMD), Rectus Femoris (RF), Lateral Hamstring (LH), Medial Hamstring (MH), Vastus Medialis (VM), Vastus Lateralis (VL), Lateral Gastrocnemius (LGS), Peroneus Longus (PL), Peroneus Brevis (PB), Soleus (SOL), and Tibialis Anterior (TA).

These signals were acquired at a sampling rate of $1000 \mathrm{~Hz}$.

The foot-switch sensor was placed beneath the first metatarsal head of the non-dominant foot (corresponding to the

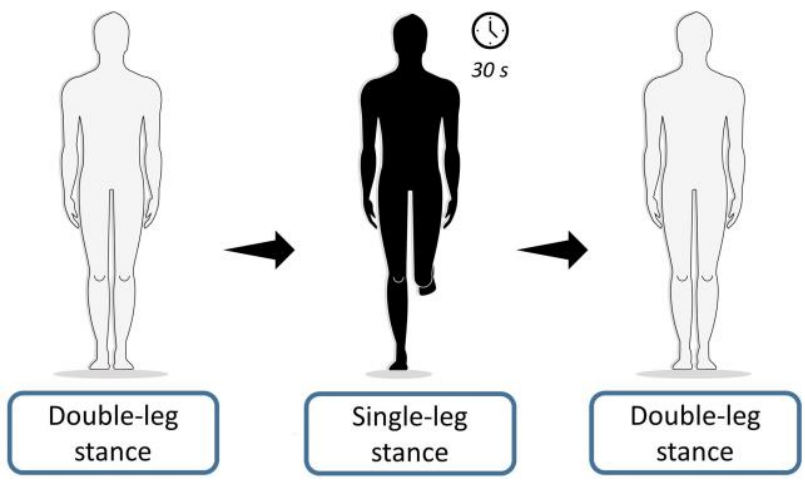

Fig. 1. Block diagram of the experimental protocol. Participants were asked to perform a transition from double-leg stance (DLS) to single-leg stance (SLS), maintaining SLS for at least 30 seconds, and then returning back to DLS.

left foot, for each subject of the sample population).

All the acquired signals were then imported into MATLAB ${ }^{\circledR}$ release R2019b (The MathWorks Inc., Natick, MA, USA) to be offline processed through custom routines.

Figure 2 describes the acquisition system composed of the sEMG active probes placed over the trunk and dominant-side muscles, the foot-switch sensor mounted on the contralateral side to detect the onset/offset timing of SLS, and the force plate to assess body sway.

\section{Data Processing}

Before muscle synergy extraction, the acquired sEMG signals were pre-processed to select the time-instants relative to WB or UB unipedal stance (SLS), discarding DLS epochs.

a) Segmentation of Single-Leg Stance (SLS) Epochs

The segmentation of the time-instants relative to WB or UB SLS was performed considering the signals acquired from the foot-switch sensor placed under the non-dominant (left) foot and the ground reaction force acquired through the force plate.

The foot-switch signal was used to detect the time-instants when the subjects moved from DLS to SLS and vice versa. The

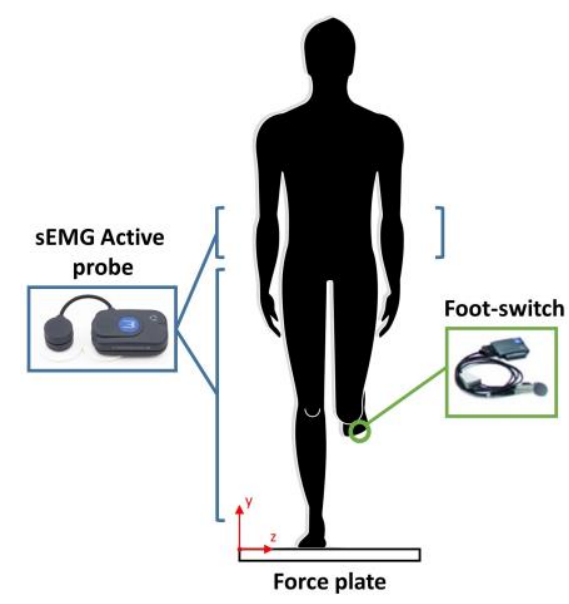

Fig. 2. Acquisition system. sEMG active probes are positioned over the main muscles of the dominant lower limb (sustaining the single-leg stance) and the trunk. A foot-switch is positioned under the first metatarsal head of the contralateral foot (raising from floor during SLS) to detect the onset/offset timing of SLS. A force plate is used to assess body sway during SLS. 
foot-switch signal was normalized in amplitude in the range $[0,1]$ :

- 0 corresponds to an open foot-switch (SLS: foot raised from the floor)

- 1 corresponds to a closed foot-switch (DLS: foo $t$ on the floor).

The onset of the SLS task was established 5 seconds after the 1-to-0 transition, while the offset was established 5 seconds before the 0-to-1 transition. In other words, we excluded from the analysis DLS-to-SLS and SLS-to-DLS transitions, keeping only the central time samples of "pure" SLS.

The ground reaction force acquired through the force plate was used to distinguish WB and UB epochs during SLS. The ground reaction force is a tri-axial signal, where the $x$-axis is aligned to the antero-posterior (AP) direction, the $y$-axis is aligned to the down-top vertical direction, and the $z$-axis is aligned to the medio-lateral (ML) direction. Due to the high correlation between the planar components (AP and ML) and the vertical component of the ground reaction force, only AP and ML were considered to separate WB from UB epochs, neglecting the vertical component of the force [1].

As a first step, each signal component was low-pass filtered through a $5^{\text {th }}$ order Butterworth digital filter with a cut-off frequency of $10 \mathrm{~Hz}$ [24], [25]. Then, the resultant force (Fres) was computed as described in (1):

$$
\text { Fres }=\sqrt{F_{A P}^{2}+F_{M L}^{2}}
$$

where $F_{A P}$ and $F_{M L}$ represent the AP and ML components of the low-pass filtered ground-reaction force, respectively.

Then, the Root-Mean-Square (RMS) of the resultant reaction force $\left(\right.$ Fres $\left._{R M S}\right)$ was computed by windowing the signal into 1s-epochs without overlap to ensure a sufficient number of samples to be used as input of the muscle synergy extraction algorithm. The time-instants belonging to WB or UB epochs were detected by applying an adaptive threshold $\left(T h_{c}\right)$ to the Fres $S_{R M S}$ signal, as described in (2):

$$
T h_{c}=\operatorname{mean}\left(\text { Fres }_{R M S}\right)+c \cdot \operatorname{std}\left(\text { Fres }_{R M S}\right)
$$

where $c$ is a multiplicative constant (of the standard deviation).

Figure 3 shows, for a representative subject, the binary mask used to separate WB from UB epochs during the SLS test (performed with eyes closed), for 3 different $c$-values $(c=0.5$, $c=1.0, c=1.5)$. These values of the constant $c$ have been chosen to achieve a sufficient length of sEMG signals for muscle synergy extraction (for both WB and UB epochs).

The binary Segmentation Mask (SM) was defined as it follows:

$$
\text { - } \quad \mathrm{SM}=1 \text {, if } \text { Fres }_{R M S} \leq T h_{c} \text { (WB epochs) }
$$$$
\text { - } \mathrm{SM}=0 \text {, if } \text { Fres }_{R M S}>T h_{c} \text { (UB epochs). }
$$

Afterwards, the sEMG signal of each muscle was segmented into WB and UB epochs using the above defined binary mask.
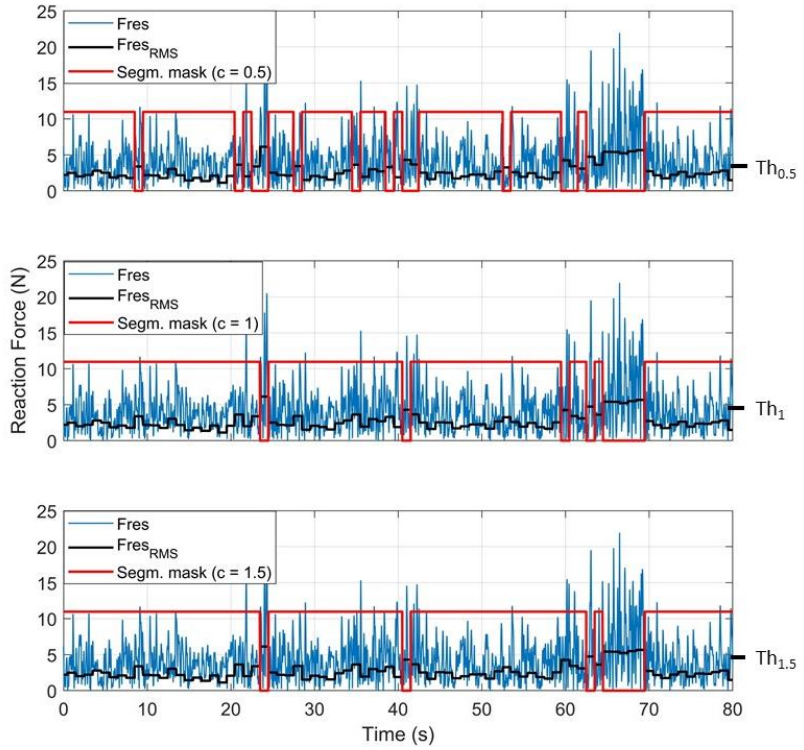

Fig. 3. Example of segmentation masks used to separate "well-balanced" and "unbalanced" single-leg stance (SLS) for a representative subject, with eyes closed, considering different values of the multiplicative constant ( $c=0.5,1.0$, and 1.5). In blue it is represented the resultant reaction force (Fres) during the SLS test, in black the Fres $_{R M S}$, while in red the segmentation mask computed considering 3 different segmentation thresholds $\left(T h_{0.5}, T h_{1.0}, T h_{1.5}\right)$. Each segmentation mask is set to 1 in correspondence of "well-balanced" SLS $\left(\right.$ Fres $\left._{R M S} \leq T h_{c}\right)$, while it is set to 0 in correspondence of excessive unipedal balance perturbations $\left(\right.$ Fres $\left._{R M S}>T h_{c}\right)$.

\section{b) Muscle Synergy Extraction and Sorting}

The segmented sEMG signals were high-pass filtered through an $8^{\text {th }}$ order Butterworth digital filter with a cut-off frequency of $35 \mathrm{~Hz}$, to remove motion artefacts, and full-wave rectified to obtain non-negative signals. The envelopes of the rectified sEMG signals were computed through a $5^{\text {th }}$ order lowpass Butterworth digital filter with a cut-off frequency of $12 \mathrm{~Hz}$ [26]. For each observed muscle, the sEMG envelopes were normalized in amplitude with respect to their global maximum to ensure equally weighted contributions of all the muscles in the muscle synergy extraction process [26].

Muscle synergies were then extracted from the amplitudenormalized sEMG envelopes by means of the Non-Negative Matrix Factorization (NNMF) algorithm. The NNMF is a widely used factorization algorithm for muscle synergy extraction [15], [27] and decomposes the original sEMG envelope matrix $(M(t))$ as the linear combination of two different components: the time-dependent activation coefficients $(C(t))$ and the time-independent weight vectors $(W)[28]$ as described in (3).

$$
M(t)=\sum_{k=1}^{N} C(t)_{k} \cdot W_{k}+e
$$

where $N$ represents the number of muscle synergies needed to model the motor control and $e$ is the reconstruction error.

The activation coefficient vector $C(t)_{k}$ represents the timedependent modulation of the muscles enrolled in the $k$-synergy (temporal component of the motor control), while the weight vector $W_{k}$ describes the time-independent contribution of each 
TABLE I

INPUT PARAMETERS OF THE MATLAB ${ }^{\circledR}$ ROUTINE “NNMF” USED FOR MUSCLE SYNERGY EXTRACTION

\begin{tabular}{lc}
\hline \hline Parameters & Values \\
\hline Algorithm & multiplicative update \\
Function tolerance & $1 \mathrm{e}^{-6}$ \\
Number of replicates & 50 \\
Number of iterations (max.) & 1000 \\
\hline \hline
\end{tabular}

muscle to the $k$-synergy (spatial component of the motor control).

The MATLAB ${ }^{\circledR}$ function "nnmf" was used to factorize the original sEMG envelope matrix, setting the routine's inputs parameters as detailed in Table I. The input parameters used in this study were optimized in previous works focused on muscle synergy extraction during gait [13], [29]. To explore different solutions of the NNMF algorithm, the "nnmf" function was run several times on the same sEMG data, changing the number of muscle synergies $(N)$ from 1 to 8 .

The reconstruction accuracy of the original sEMG envelope matrix $(M(t))$ was computed for each number of muscle synergies $(N)$ by means of the total Variance Accounted For $(t V A F)$, defined as the uncentered Pearson's correlation coefficient expressed in percentage (4):

$$
t V A F=\left(1-\frac{\sum_{k=1}^{m}\left(M_{k}-M_{k}^{R}\right)^{2}}{\sum_{k=1}^{m}\left(M_{k}\right)^{2}}\right) \cdot 100
$$

where $m$ represents the number of observed muscles, while $M_{k}^{R}$ and $M_{k}$ represent the reconstructed and the original sEMG envelopes of the $k$-muscle, respectively.

The optimal number of muscle synergies $\left(N_{\text {opt }}\right)$ needed to properly reconstruct the original sEMG matrix $(M(t))$ was selected by choosing the least number of muscle synergies ensuring $t V A F \geq 90 \%$ (global criterion) [30]. Moreover, considering the number of muscle synergies selected according to the above criterion, the Variance Accounted For $(V A F)$ was also computed for each of the observed muscles. If $V A F \geq 75 \%$ for each of the 13 muscles (local criterion), it was concluded that no additional muscle synergies were needed to reconstruct the original sEMG envelopes. Otherwise $(V A F<75 \%)$, the number of muscle synergies $(N)$ was incremented until all the muscles achieved a $V A F$ value equal to or greater than $75 \%$ [16], [31].

To graphically represent the muscle synergies, the weight vectors $(W)$ were normalized in amplitude in the range $[0,1]$ with respect to their global maximum. Then, the activation coefficient vectors $(C(t))$ were multiplied by the correspondent normalized values.

To sort the muscle synergies in the same order for each subject and condition, a $k$-means clustering algorithm was applied to the weight vectors $(W)$ [32]. The clustering algorithm was set considering $N_{\text {opt }}$ as number of $k$-means clusters, 1000 as maximum number of iterations, 15 as number of replicates, and cosine similarity as distance metric. The activation coefficients $(C(t))$ were then sorted consequently.

\section{E. Robustness of the Segmentation Threshold}

To assess if the selection of both WB and UB epochs of SLS is robust with respect to the segmentation threshold $T h_{c}$, we used the following procedure. Separately for WB and UB epochs, we compared the muscle synergies extracted from sEMG envelopes, considering 3 different values of the constant $c$ defined in (2): $c=0.5, c=1.0$, and $c=1.5$.

The muscle synergies extracted using $T h_{0.5}, T h_{1.0}$, and $T h_{1.5}$ were quantitatively compared in terms of the consistency of the optimal number of muscle synergies $\left(N_{\text {opt }}\right)$, and the similarity of weight vectors estimated through Pearson's correlation coefficient $(R)$.

\section{F. "Well-balanced" and "Unbalanced" Single-Leg Stance}

To justify the necessity to distinguish between WB and UB epochs of SLS, the correspondent muscle synergies were extracted (setting $c=1.0$ ) and compared in terms of $(a)$ the optimal number of muscle synergies $\left(N_{\text {opt }}\right),(b)$ the average recruitment level of the activation coefficient vectors (Recr), and $(c)$ the balance control strategies $(S)$.

\section{a) Optimal Number of Muscle Synergies ( $N_{\text {opt }}$ )}

As stated before, the optimal number of muscle synergies $\left(N_{\text {opt }}\right)$ necessary to properly reconstruct the original sEMG envelopes was selected by choosing the smallest number of synergies which guarantees $t V A F \geq 90 \%$ (global criterion) and $V A F \geq 75 \%$ (local criterion) for each of the observed muscles [16], [30], [31].

\section{b) Average Recruitment Level (Recr)}

Since in SLS balance control there are no typical cyclostationary processes, any direct interpretation of the activation coefficient vectors $C(t)_{k}$ is difficult. Therefore, only the average recruitment level was considered to quantitatively compare muscle synergy activation coefficient vectors $(C(t))$ [27]. The average recruitment level of the $k$-synergy $\left(\operatorname{Recr}_{k}\right)$ was computed as the average (over time) of the activation coefficient vector $C(t)_{k}$.

\section{c) Balance Control Strategy (S)}

Considering the task performed and the acquired muscles, three different balance control strategies can be identified: (i) ankle control, (ii) knee control, and (iii) hip/trunk control [19].

i. The ankle control strategy $\left(S_{\text {ankle }}\right)$ is mainly related to the activation of 5 muscles of the leg: PL, PB, TA, LGS, and SOL.

ii. The knee control strategy $\left(S_{\text {knee }}\right)$ is mainly related to the activation of 3 muscles of shank: VM, VL, and RF.

iii. The hip/trunk control strategy $\left(S_{\text {hip }}\right)$ is mainly related to the activation of 5 muscles of the proximal lower limb and the trunk: $\mathrm{LH}, \mathrm{MH}, \mathrm{GMD}, \mathrm{LD}_{\mathrm{R}}$, and $\mathrm{LD}_{\mathrm{L}}$. 
The balance control strategies were quantified by computing the average weight vector $\left(W_{k}\right)$ across those muscles that belong to the same balance control strategy in the $k$-synergy, as detailed in (5):

$$
S_{j, k}=\frac{\sum_{i=1}^{m} W_{k, i}}{m}
$$

where $S_{j, k}$ represents the $j$-th balance control strategy ( $j=1$ : ankle; 2: knee; 3: hip) for the $k$-synergy, and $m$ represents the number of muscles enrolled in the $j$-th balance control strategy.

A single balance control strategy $\left(S_{k}\right)$ was then associated to each muscle synergy by calculating the highest $S_{j, k}$ among those computed for the same $k$-synergy, as detailed in (6).

$$
S_{k}=\max \left(S_{1 k}, S_{2 k}, S_{3 k}\right)
$$

where $k$ can assume value from 1 to $3\left(S_{1}=S_{\text {ankle }}, S_{2}=S_{\text {knee }}\right.$, $\left.\mathrm{S}_{3}=\mathrm{S}_{\text {hip }}\right)$.

\section{G. Eyes Open and Eyes Closed Conditions}

To assess the impact of the visual feedback in maintaining SLS, muscle synergies were separately extracted from the sEMG envelopes in correspondence of the EO and EC conditions and then quantitatively compared considering the same parameters detailed in the previous section.

\section{H. Statistical Analysis}

A two-way analysis of variance (ANOVA) followed by posthoc analysis with Bonferroni adjustment for multiple comparisons was performed to assess the robustness of the segmentation threshold.

To assess significant changes in the optimal number of muscle synergies, in the average recruitment levels, and in the balance control strategies considering different sEMG epochs (WB and UB) and during different SLS conditions (EO and EC), firstly the hypothesis of normality of the distribution was tested using the Lilliefors test with a significance level $(\alpha)$ of 0.05 . If the normality hypothesis was rejected, the Wilcoxon signed-rank test $(\alpha=0.05)$ was performed, otherwise a twotailed paired Student's $t$-test was performed $(\alpha=0.05)$.

\section{RESULTS}

On average, the number of WB and UB SLS epochs were similar ( $8 \pm 3 \mathrm{WB} / \mathrm{UB}$ epochs), but different epoch durations were measured. During the EO condition, the average WB and UB epoch durations were equal to $70.5 \mathrm{~s} \pm 9.8 \mathrm{~s}$ and $10.3 \mathrm{~s} \pm$ $2.9 \mathrm{~s}$, respectively, while considering the EC condition they were equal to $42.8 \mathrm{~s} \pm 27.3 \mathrm{~s}$ and $5.8 \mathrm{~s} \pm 3.8 \mathrm{~s}$.

First, we present the results related to the robustness of the segmentation threshold. Secondly, we present the results that justify the separation into WB and UB epochs of SLS. Finally, the muscle synergies obtained considering the two different SLS conditions (EO and EC) are quantitatively compared, separately, for WB and UB epochs.

\section{a) Robustness of the Segmentation Threshold}

All the tested segmentation thresholds $\left(T h_{0.5}, T h_{1.0}\right.$, and $\left.T h_{1.5}\right)$ required the same number of muscle synergies $\left(N_{\text {opt }}\right)$ to properly reconstruct the original sEMG envelopes $(t V A F \geq$ $90 \%$ and $V A F \geq 75 \%$ for each muscle). More specifically, considering WB epochs, for every threshold 4 muscle synergies were needed to reconstruct the SEMG data, both in EO and EC conditions. The same results were obtained considering UB epochs.

Moreover, results revealed high values of the Pearson's correlation coefficients between each pair of thresholds. For $\mathrm{WB}$, we found no statistically significant differences in terms of weight-vector correlation among the 3 thresholds $(p=0.17)$ and the 2 tested conditions $(p=0.87)$. For UB, we found no statistically significant differences among thresholds $(p=0.28)$, while a significant decrease $(p=0.01)$ in the weight correlation was detected in the EO condition with respect to the EC condition.

Table II shows the Pearson's correlation coefficients $(R)$, averaged on the sample population, between each pair of thresholds, separately for WB and UB epochs. Results suggest a very high similarity among the muscle synergy weight vectors extracted considering the 3 thresholds, both in WB and UB epochs.

Considering the high similarity of the weight vectors and the high consistency of the optimal number of muscle synergies obtained from the 3 thresholds, the multiplicative constant $c$ of the segmentation threshold was set equal to 1.0.

\section{b) "Well-balanced" and "Unbalanced" Single-Leg Stance}

The muscle synergies were extracted from the sEMG envelopes in correspondence of WB and UB epochs of SLS, to justify the segmentation process.

No significant differences were found in terms of the number of muscle synergies between WB and UB epochs. In particular, considering the EO condition, 4 muscle synergies were necessary to reconstruct the original sEMG data with a $t V A F$ value of $93.0 \% \pm 1.2 \%$ for $\mathrm{WB}$, and $93.1 \% \pm 1.3 \%$ for $\mathrm{UB}$, respectively. Similar results were obtained considering the EC condition, where 4 muscle synergies were extracted with a $t V A F$ value of $92.6 \% \pm 1.5 \%$ for $\mathrm{WB}$, and $92.8 \% \pm 1.3 \%$, for $\mathrm{UB}$, respectively.

Figure 4 shows the muscle synergies, averaged over the sample population, extracted from the 2 different conditions: EO in Figure 4A, and EC in Figure 4B, respectively. For each condition, the muscle synergies extracted considering WB and

TABLE II

WEIGHT VECTOR CORRELATION $(R)$ AVERAGED ON THE SAMPLE POPULATION

\begin{tabular}{ccccc}
\hline \hline \multirow{2}{*}{ SLS epochs } & \multicolumn{3}{c}{$\begin{array}{c}\text { Pearson's Correlation Coefficient }(R) \\
\text { (mean } \pm \text { standard deviation) }\end{array}$} \\
\cline { 3 - 5 } & & $T h_{0.5} v s T h_{1}$ & $T h_{0.5} v s T h_{1.5}$ & $T h_{1} v s T h_{1.5}$ \\
\hline Well-balanced & EO & $0.91 \pm 0.16$ & $0.92 \pm 0.14$ & $0.95 \pm 0.11$ \\
& EC & $0.91 \pm 0.15$ & $0.89 \pm 0.17$ & $0.97 \pm 0.08$ \\
Unbalanced & EO & $0.84 \pm 0.19$ & $0.75 \pm 0.20$ & $0.80 \pm 0.20$ \\
& EC & $0.88 \pm 0.17$ & $0.86 \pm 0.17$ & $0.90 \pm 0.18$ \\
\hline \hline
\end{tabular}

Th..5: first threshold with $c=0.5 ; T h_{1.0}$ : second threshold with $c=1.0 ; T h_{1.5}$ : third threshold with $c=1.5$. EO: Eyes Open; EC: Eyes Closed. 


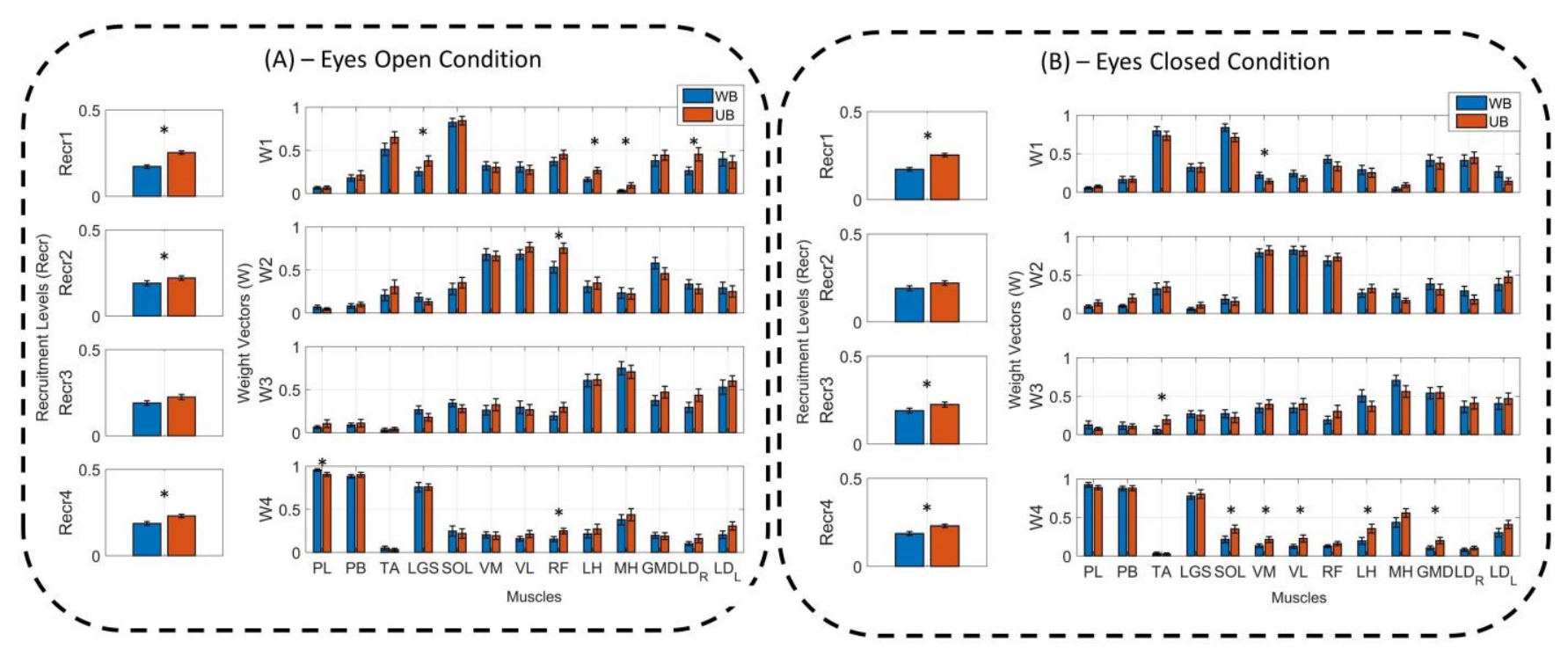

Fig. 4. Comparison of the muscle synergies extracted during "well-balanced" (WB) and "unbalanced" (UB) epochs of single-leg stance (SLS), for both the eyes open (EO) and eyes closed (EC) conditions. In both panel (A) and (B), the colored vertical bars represent the average recruitment levels $R_{e c r}$ (on the left) and weight vectors $W_{k}$ (on the right) of the $k$-synergy, over the sample population, with the superimposition of the standard error (black lines). The asterisk $(*)$ indicates a statistically significant difference $(p<0.05)$.

UB epochs of SLS are compared. In particular, for each muscle synergy, the average recruitment level Recr $_{k}$ and weigh vector $W_{k}$ are reported.

We found a statistically significant increase in the recruitment levels of three out of four muscle synergies extracted considering UB epochs with respect to those extracted considering WB epochs, during both EO and EC conditions. The values of the average recruitment levels of each muscle synergy are presented in Table III, as well as the indication of the statistically significant changes between $\mathrm{WB}$ and UB epochs (indicated by asterisks).

Figure 4 (A and B) shows significant changes $(p<0.05)$ of the average recruitment levels as well as the contribution of the observed muscles to each muscle synergy in EO and EC conditions.

The first and the fourth muscle synergies can be mainly associated to an ankle control strategy, the second muscle synergy to a knee control strategy, and the third muscle synergy to a hip/trunk control strategy. Considering the EO condition, results revealed a statistically significant increase of the ankle $(p=0.05)$ control strategy recruitment in the UB epochs with respect to the WB ones. No statistically significant changes were found evaluating the knee and hip/trunk balance control strategy associated to the third muscle synergy between WB and UB epochs. Considering the EC condition, instead, no statistically significant changes of the balance control strategies were observed between WB and UB epochs. The values of the average balance control strategies are presented in Table IV with the indication of the statistically significant changes between WB and UB epochs of SLS (indicated by asterisks).

Therefore, we demonstrated that the muscle synergies extracted considering WB and UB epochs during SLS are different, both in terms of average recruitment level and balance control strategy. This justifies the segmentation process as a necessary pre-processing procedure to properly assess the motor control strategies and to help the interpretation of the muscle synergies during SLS.

\section{c) Eyes Open and Eyes Closed Conditions}

In the following, muscle synergy results between EO and EC conditions are compared (considering separately WB and UB epochs of SLS).

As already mentioned in the previous section, both EO and EC conditions required the same number of muscle synergies $\left(N_{o p t}\right)$ to properly reconstruct the original sEMG envelopes (for both WB and UB epochs).

Figure 5 reports the muscle synergies, averaged over the sample population, extracted from the WB (Figure 5A) and UB (Figure 5B) epochs. Considering each SLS epoch (Figure 5A or Figure 5B), the muscle synergies extracted during EO and EC conditions are directly compared.

A statistically significant increase in the recruitment levels of the fourth muscle synergy (Recr $\left.{ }_{4}\right)$ was found in the EC condition with respect to the EO condition, for both WB ( $p=$ $0.03)$ and UB $(p<0.0001)$ epochs. No other statistically significant changes were found on the recruitment level of the remaining muscle synergies, comparing EO and EC conditions. The values of the average recruitment levels are presented in Table III with the indication of the statistically significant changes between EO and EC conditions (indicated by daggers).

Considering WB epochs, we found a statistically significant increase of the ankle $(p=0.02)$ and knee $(p=0.03)$ control strategy recruitment in the EC condition with respect to the EO condition. No statistically significant changes were found on the hip/trunk balance control strategy between the EO and EC conditions. 


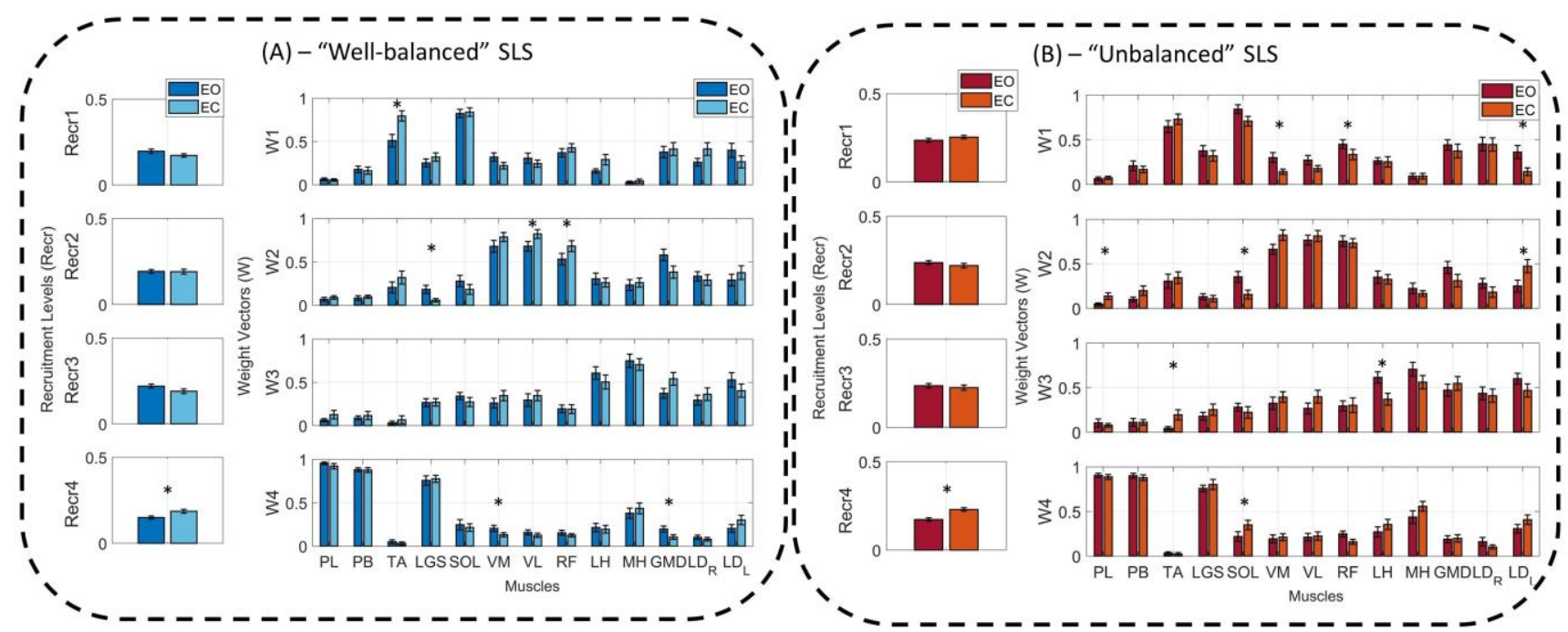

Fig. 5. Comparison of the muscle synergies extracted during eyes open (EO) and eyes closed (EC) conditions, for both "well-balanced" and "unbalanced" epochs of single-leg stance (SLS). In both panel (A) and (B), colored vertical bars represent the average recruitment levels $\operatorname{Recr}_{k}$ (on the left) and weight vectors $W_{k}$ (on the right) of the $k$-synergy, over the sample population, with the superimposition of the standard error (black lines). The asterisk (*) indicates a statistically significant difference $(p<0.05)$.

Considering UB epochs, instead, no statistically significant changes in the balance control strategies were assessed between the EO and EC conditions. The values of the average balance control strategies are presented in Table IV with the indication of the statistically significant changes between EO and EC conditions (indicated by daggers).

\section{DisCUSSION AND CONCLUSIONS}

The muscle synergy hypothesis is a well-known approach used in literature to assess the modular organization of the central nervous system during different motor tasks, such as single-leg stance. In the last years, the study of muscle synergies during SLS was mainly focused on the evaluation of balance recovery after a perturbation [17]-[21], rather than on the maintenance of the balance condition itself. The methodology proposed in this paper can be used to assess the motor control strategies adopted to maintain SLS,

TABLE III

RECRUITMENT LEVELS (Recr) AVERAGED ON THE SAMPLE POPULATION

\begin{tabular}{lccc}
\hline \hline & & \multicolumn{2}{c}{$\begin{array}{c}\text { Average Recruitment Levels (Recr) } \\
\text { (mean } \pm \text { standard deviation) }\end{array}$} \\
\cline { 2 - 4 } Recruitment Levels & $\begin{array}{c}\text { Well-balanced } \\
(\text { WB })\end{array}$ & $\begin{array}{c}\text { Unbalanced } \\
(\text { UB })\end{array}$ \\
\hline \multirow{2}{*}{ EO } & Recr $_{1}$ & $0.20 \pm 0.06^{*}$ & $0.24 \pm 0.05^{*}$ \\
& Recr $_{2}$ & $0.19 \pm 0.05^{* *}$ & $0.24 \pm 0.05^{* *}$ \\
& Recr $_{3}$ & $0.22 \pm 0.06$ & $0.24 \pm 0.06$ \\
& Recr $_{4}$ & $0.15 \pm 0.04^{* \dagger}$ & $0.17 \pm 0.05^{* *}$ \\
\multirow{4}{*}{ EC } & Recr $_{1}$ & $0.17 \pm 0.05^{* * *}$ & $0.25 \pm 0.05^{* * *}$ \\
& Recr $_{2}$ & $0.19 \pm 0.07$ & $0.22 \pm 0.06$ \\
& Recr $_{3}$ & $0.19 \pm 0.06^{*}$ & $0.22 \pm 0.07^{*}$ \\
& Recr $_{4}$ & $0.19 \pm 0.05^{* * *}$ & $0.23 \pm 0.05^{* * *}$ \\
\hline \hline
\end{tabular}

$\operatorname{Recr}_{\mathrm{k}}$ represents the average recruitment level of the $k$-synergy $(k=1,2,3,4)$. The asterisk $(*)$ indicates a statistically significant difference ( $p<0.05$ ) between well-balanced (WB) and unbalanced (UB) epochs of single-leg stance (SLS), while the dagger ( $\dagger$ ) between eyes open (EO) and eyes closed (EC) conditions. Single, double, and triple asterisks (or daggers) represent $p$-values lower than $0.05,0.01$, and 0.001 , respectively. distinguishing between epochs of sEMG signals relative to a "well-balanced" (WB) and "unbalanced" (UB) SLS.

The consistency of the optimal number of muscle synergies and the high similarity of the weight vectors across different values of the adaptive segmentation threshold suggest that the proposed approach is robust (the weight vector correlation coefficient $\mathrm{R}$ ranges from 0.75 to 0.97 ). The 4 muscle synergies extracted from WB and UB sEMG epochs are in line with previous studies [19], [27], in which similar muscle synergies were needed to accurately assess the balance control strategies during SLS after the application of multidirectional perturbations. However, a fewer number of muscle synergies was computed in this contribution with respect to the previous ones due to the reduced complexity of the balance task analyzed.

By considering the number and the composition of the muscle synergies during WB- and UB-SLS, no statistically significant differences were detected. However, differences in terms of average recruitment levels and balance control strategies suggest a slightly different modular organization

TABLE IV

BALANCE CONTROL STRATEGIES $(S)$ AVERAGED ON THE SAMPLE POPULATION

\begin{tabular}{cccc}
\hline \hline & & \multicolumn{2}{c}{$\begin{array}{c}\text { Average Balance Control Strategies }(S) \\
\text { (mean } \pm \text { standard deviation) }\end{array}$} \\
\cline { 3 - 4 } $\begin{array}{c}\text { Balance Control } \\
\text { Strategies }\end{array}$ & $\begin{array}{c}\text { Well-balanced } \\
(\boldsymbol{W B})\end{array}$ & $\begin{array}{c}\text { Unbalanced } \\
(\text { UB })\end{array}$ \\
\hline \multirow{2}{*}{ EO } & Ankle & $0.37 \pm 0.11^{* \dagger}$ & $0.43 \pm 0.11^{*}$ \\
& Knee & $0.63 \pm 0.24^{\dagger}$ & $0.73 \pm 0.22$ \\
& Hip/Trunk & $0.51 \pm 0.14$ & $0.56 \pm 0.16$ \\
\multirow{2}{*}{ EC } & Ankle & $0.43 \pm 0.12^{\dagger}$ & $0.40 \pm 0.12$ \\
& Knee & $0.76 \pm 0.20^{\dagger}$ & $0.79 \pm 0.24$ \\
& Hip/Trunk & $0.50 \pm 0.20$ & $0.49 \pm 0.08$ \\
\hline \hline
\end{tabular}

Average balance controls of each of the three identified strategies. The asterisk (*) indicates a statistically significant difference $(p<0.05)$ between well-balanced (WB) and unbalanced (UB) epochs of single-leg stance (SLS), while the dagger $(\dagger)$ between eves open (EO) and eyes closed (EC) conditions. Single, double, and triple asterisks (or daggers) represent $p$-values lower than $0.05,0.01$, and 0.001 , respectively. 
between WB- and UB-SLS epochs. More specifically, UB-SLS epochs require higher average recruitment levels, in both EO and EC conditions, and an increased ankle control strategy, in the EO condition. These differences justify the necessity to distinguish between WB and UB unipedal-stance epochs of sEMG signal, when feeding the algorithm for muscle synergy extraction. Our results are consistent with the findings of previous studies in which the effect of the task complexity, postural configuration, and loading conditions on motor control strategies were assessed [19], [22], [23]. Results suggest that, for a specific balance task, our central nervous system recruits similar muscle synergies rather than generating completely new set of muscle synergies for each task condition. Indeed, we found only slight modifications to the basic motor control strategies involved in SLS, while differences in terms of average recruitment levels and balance control strategies were assessed between EO and EC conditions. More specifically, the $\mathrm{EC}$ condition requires a higher average recruitment level of the fourth muscle synergy (considering both WB- and UB-SLS epochs), and an increased level of ankle and knee control strategies (considering WB-SLS epochs), suggesting a higher recruitment of the muscle synergies controlling the distal muscles.

This study was conducted on young healthy subjects that are able to maintain equilibrium on one foot, even with their eyes closed, for at least $30 \mathrm{~s}$. However, it may be difficult to apply this same protocol to elderly or pathological populations affected by severe balance impairments. The finding that the muscle synergies are, overall, similar between WB and UB epochs, can depend on the specific population considered. Different results might be obtained in populations with diminished equilibrium skills.

Another limitation of this study is that it focused only on balance strategies during SLS maintenance, without analyzing transition tasks. The analyzed signals started 5 seconds after the first (DLS-to-SLS) transition, and stopped 5 seconds before the second (SLS-to-DLS) transition. Therefore, our findings on motor control strategies adopted during SLS (excluding the transitions) cannot be extended to task transitions. Future studies might analyze this important aspect [33].

In conclusion, our results demonstrate that the proposed approach for the selection of sEMG epochs relative to "wellbalanced" and "unbalanced" SLS is robust with respect to the selection of the segmentation threshold and can be successfully used as a pre-processing step before muscle synergy extraction, allowing a better assessment of motor control strategies during the maintenance of the single-leg stance. Further studies will focus on the application of this approach to sEMG signals acquired from subjects affected by chronic ankle instability (CAI) during SLS task, to assess its applicability in pathological conditions.

\section{REFERENCES}

M. Ghislieri, L. Gastaldi, S. Pastorelli, S. Tadano, and V. Agostini, "Wearable Inertial Sensors to Assess Standing Balance: A Systematic Review," Sensors, vol. 19, no. 19, p. 4075, Sep. 2019, doi: 10.3390/s19194075 chronic ankle instability exhibit decreased postural sway while kicking in a single-leg stance," Gait Posture, vol. 40, no. 1, pp. 231236, May 2014, doi: 10.1016/j.gaitpost.2014.04.002.

[3] S. Van Deun, F. F. Staes, K. H. Stappaerts, L. Janssens, O. Levin, and K. K. H. Peers, "Relationship of chronic ankle instability to muscle activation patterns during the transition from double-leg to single-leg stance," Am. J. Sports Med., vol. 35, no. 2, pp. 274-281, 2007, doi: $10.1177 / 0363546506294470$.

[4] M. Pope, L. Chinn, D. Mullineaux, P. O. McKeon, L. Drewes, and J. Hertel, "Spatial postural control alterations with chronic ankle instability," Gait Posture, vol. 34, no. 2, pp. 154-158, 2011, doi: https://doi.org/10.1016/j.gaitpost.2011.04.012.

[5] V. Agostini, E. Chiaramello, C. Bredariol, C. Cavallini, and M. Knaflitz, "Postural control after traumatic brain injury in patients with neuro-ophthalmic deficits.," Gait Posture, vol. 34, no. 2, pp. 248253, 2011, doi: 10.1016/j.gaitpost.2011.05.008.

[6] V. Agostini, A. Sbrollini, C. Cavallini, A. Busso, G. Pignata, and M. Knaflitz, "The role of central vision in posture: Postural sway adaptations in Stargardt patients," Gait Posture, Oct. 2015, doi: 10.1016/j.gaitpost.2015.10.003.

[7] D. Barbado Murillo, R. Sabido Solana, F. J. Vera-Garcia, N. Gusi Fuertes, and F. J. Moreno, "Effect of increasing difficulty in standing balance tasks with visual feedback on postural sway and EMG: Complexity and performance," Hum. Mov. Sci., vol. 31, no. 5, pp. 1224-1237, 2012, doi: https://doi.org/10.1016/j.humov.2012.01.002.

[8] P. Balestrucci, E. Daprati, F. Lacquaniti, and V. Maffei, "Effects of visual motion consistent or inconsistent with gravity on postural sway," Exp. Brain Res., vol. 235, no. 7, pp. 1999-2010, 2017, doi: 10.1007/s00221-017-4942-3.

[9] L. H. Ting and J. L. McKay, "Neuromechanics of muscle synergies for posture and movement," Curr Opin Neurol, vol. 17, no. 6, pp. 622-628, 2007, doi: 10.1016/j.conb.2008.01.002.

[10] S. A. Chvatal and L. H. Ting, "Common muscle synergies for balance and walking," Front. Comput. Neurosci., vol. 7, p. 48, May 2013, doi: 10.3389/fncom.2013.00048.

[11] D. Rimini, V. Agostini, and M. Knaflitz, "Intra-Subject Consistency during Locomotion: Similarity in Shared and Subject-Specific Muscle Synergies," vol. 11, no. December, pp. 1-10, 2017, doi: 10.3389/fnhum.2017.00586.

[12] D. Rimini, V. Agostini, and M. Knaflitz, "Evaluation of muscle synergies stability in human locomotion: A comparison between normal and fast walking speed," I2MTC 2017 - 2017 IEEE Int. Instrum. Meas. Technol. Conf. Proc., no. June 2019, 2017, doi: 10.1109/I2MTC.2017.7969722.

[13] M. Ghislieri, V. Agostini, and M. Knaflitz, "Muscle Synergies Extracted Using Principal Activations: Improvement of Robustness and Interpretability," IEEE Trans. Neural Syst. Rehabil. Eng., pp. 11, 2020, doi: 10.1109/TNSRE.2020.2965179.

[14] J. Taborri et al., "Feasibility of Muscle Synergy Outcomes in Clinics, Robotics, and Sports: A Systematic Review," Appl. Bionics Biomech., vol. 2018, pp. 1-19, Apr. 2018, doi: $10.1155 / 2018 / 3934698$.

[15] D. D. Lee and H. S. Seung, "Learning the parts of objects by nonnegative matrix factorization.," Nature, vol. 401, no. 6755, pp. 788791, 1999, doi: 10.1038/44565.

[16] G. Torres-Oviedo and L. H. Ting, "Muscle Synergies Characterizing Human Postural Responses," J. Neurophysiol., vol. 98, no. 4, pp. 2144-2156, 2007, doi: 10.1152/jn.01360.2006.

[17] V. Munoz-Martel, A. Santuz, A. Ekizos, and A. Arampatzis, "Neuromuscular organisation and robustness of postural control in the presence of perturbations," Sci. Rep., vol. 9, no. 1, p. 12273, Dec. 2019, doi: 10.1038/s41598-019-47613-7.

[18] M. Yamagata, A. Falaki, and M. L. Latash, "Stability of vertical posture explored with unexpected mechanical perturbations: synergy indices and motor equivalence," Exp. Brain Res., vol. 236, no. 5, pp. 1501-1517, 2018, doi: 10.1007/s00221-018-5239-x.

[19] G. Torres-Oviedo and L. H. Ting, "Subject-Specific Muscle Synergies in Human Balance Control Are Consistent Across Different Biomechanical Contexts," J Neurophysiol, vol. 103, no. 6, pp. 3084-3098, 2010, doi: 10.1152/jn.00960.2009.

[20] T. Wojtara, F. Alnajjar, S. Shimoda, and H. Kimura, "Muscle synergy stability and human balance maintenance," J Neuroeng Rehabil, vol. 11, p. 129, 2014, doi: 10.1186/1743-0003-11-129.

[21] P. A. Burtner, C. Qualls, and M. H. Woollacott, "Muscle activation characteristics of stance balance control in children with spastic 
cerebral palsy," Gait Posture, vol. 8, no. 3, pp. 163-174, 1998, doi: https://doi.org/10.1016/S0966-6362(98)00032-0.

[22] A. D'Avella and E. Bizzi, "Shared and specific muscle synergies in natural motor behaviors," Proc. Natl. Acad. Sci. U. S. A., vol. 102, no. 8, pp. 3076-3081, 2005, doi: 10.1073/pnas.0500199102.

[23] V. C. K. Cheung, A. D'Avella, and E. Bizzi, "Adjustments of motor pattern for load compensation via modulated activations of muscle synergies during natural behaviors.," J Neurophysiol, vol. 101, no. 3, pp. 1235-1257, 2009, doi: 10.1152/jn.01387.2007.

[24] K. L. Hsieh, K. L. Roach, D. A. Wajda, and J. J. Sosnoff, "Smartphone technology can measure postural stability and discriminate fall risk in older adults.," Gait Posture, vol. 67, pp. 160165, Jan. 2019, doi: 10.1016/j.gaitpost.2018.10.005.

[25] M. Mancini, F. B. Horak, C. Zampieri, P. Carlson-Kuhta, J. G. Nutt, and L. Chiari, "Trunk accelerometry reveals postural instability in untreated Parkinson's disease," Parkinsonism Relat. Disord., vol. 17, no. 7, pp. 557-562, Aug. 2011, doi: 10.1016/J.PARKRELDIS.2011.05.010.

[26] D. Torricelli et al., "Muscle Synergies in Clinical Practice: Theoretical and Practical Implications," vol. 10, pp. 251-272, 2016, doi: 10.1007/978-3-319-24901-8.

[27] G. Torres-Oviedo and L. H. Ting, "Muscle synergies characterizing human postural responses," J Neurophysiol, vol. 98, no. 4, pp. 21442156, 2007, doi: 10.1152/jn.01360.2006.

[28] K. E. Zelik, V. La Scaleia, Y. P. Ivanenko, and F. Lacquaniti, "Can modular strategies simplify neural control of multidirectional human locomotion?," J Neurophysiol, vol. 111, no. 8, pp. 1686-1702, 2014, doi: $10.1152 /$ jn.00776.2013.

[29] M. Ghislieri, V. Agostini, and M. Knaflitz, "How to Improve Robustness in Muscle Synergy Extraction," in 2019 41st Annual International Conference of the IEEE Engineering in Medicine and Biology Society (EMBC), Jul. 2019, pp. 1525-1528, doi: 10.1109/EMBC.2019.8856438.

[30] D. J. Clark, L. H. Ting, F. E. Zajac, R. R. Neptune, and S. A. Kautz, "Merging of healthy motor modules predicts reduced locomotor performance and muscle coordination complexity post-stroke.," $J$ Neurophysiol, vol. 103, no. 2, pp. 844-857, 2010, doi: 10.1152/jn.00825.2009.

[31] L. H. Ting and S. A. Chvatal, "Motor control: theories, experiments, and applications," pp. 102-138, 2010.

[32] K. M. Steele, M. C. Tresch, and E. J. Perreault, "Consequences of biomechanically constrained tasks in the design and interpretation of synergy analyses.," J. Neurophysiol., vol. 113, no. January, p. jn.00769.2013, 2015, doi: 10.1152/jn.00769.2013.

[33] B. Dingenen, F. F. Staes, and L. Janssens, "A new method to analyze postural stability during a transition task from double-leg stance to single-leg stance," J. Biomech., vol. 46, no. 13, pp. 2213-2219, 2013, doi: 10.1016/j.jbiomech.2013.06.026. 